\title{
Surgical considerations for tremor and dystonia
}

\section{ABSTRACT}

Deep brain stimulation (DBS) is among the most effective approaches for the treatment of patients with advanced movement disorders. In patients with essential tremor, stimulation typically targets the ventral intermediate nucleus of the thalamus. Results of several studies have shown that over a follow-up period of 1 to 5 years, the severity of tremor decreases by an average of approximately $50 \%$ from baseline. Ongoing research continues to define the optimal stimulation parameters for patients with tremor, including frequency, voltage, and pulse width. In patients with dystonia, DBS typically targets the globus pallidus internus or the subthalamic nucleus. Longterm prospective clinical trials demonstrated reductions in motor severity rating scale scores of approximately $50 \%$ to $80 \%$ over follow-up periods of 2 to 3 years. Serious adverse events were uncommon, and included lead failures and infections. Appropriate candidates for DBS treatment of dystonia include patients with an unequivocal diagnosis of dystonia and significant disability. Several issues in the use of DBS for movement disorders remain unresolved, including the intensity of appropriate medical management before undergoing DBS, the importance of intraoperative mapping, optimal stimulator programming, and the time course of the beneficial effects of treatment.

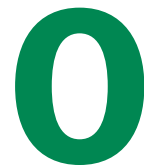

ver the last decade, several studies have demonstrated that deep brain stimulation (DBS) is among the most effective approaches for the treatment of patients with advanced movement disorders, including chorea, levodopa-induced dyskinesia, tremor, and dystonia. ${ }^{1}$ The goal of DBS is to restore function or relieve pain by stimulating neuronal activity through surgically implanted electrodes. DBS

Both authors reported that they have no financial interests or relationships that pose a potential conflict of interest with this article.

This article is based on Dr. Cooper's presentation at "The Annual Therapy Symposium on Movement Disorders for the Modern Clinician" held in Fort Lauderdale, Florida, on January 29, 2011. The article was drafted by Cleveland Clinic Journal of Medicine staff, including Mark Bowes, PhD, and was then reviewed, revised, and approved by Dr. Cooper.

doi:10.3949/ccjm.79.s2a.08 produces marked and persistent reductions in abnormal movements in patients with common hyperkinetic disorders, with a generally low incidence of serious adverse events in pediatric patients and adults.

\section{DEEP BRAIN STIMULATION FOR ESSENTIAL TREMOR}

Tremor is a rhythmic, involuntary, oscillatory movement of a body part. Tremors may be subdivided into several categories on the basis of clinical signs and symptoms, including rest, postural, and kinetic. ${ }^{2}$ Essential tremor is the most common tremor disorder, affecting an estimated $5 \%$ of the population over the age of 60 years. ${ }^{3}$ Tremor is also commonly associated with other neurologic conditions, including multiple sclerosis, Parkinson disease, and severe head trauma. ${ }^{3}$ Hand, head, and vocal tremor are the most common clinical manifestations of essential tremor, and may significantly interfere with normal function. ${ }^{4}$ For example, the effect of essential tremor on a simple hand-drawing task is illustrated in Figure 1, which demonstrates the marked tremor-related impairment in a patient's ability to draw a spiral shape and the resulting improvement in hand coordination after the application of DBS.

\section{Improvement with thalamic DBS}

The ventral intermediate nucleus (VIM) of the thalamus is the most common target for DBS treatment of essential tremor. Several studies have demonstrated significant long-term improvement in tremor following thalamic DBS. ${ }^{3}$ Most studies enrolled 20 to 30 patients, who were followed for 1 to 5 years after device implantation. On average, these studies reported an improvement in overall tremor of approximately 50\% from baseline with thalamic DBS.

\section{Patient selection and stimulation parameters}

Symptoms targeted for DBS treatment include unilateral and sometimes bilateral limb tremor. Some evidence exists for effectiveness in axial and vocal tremor as well. Factors to consider in patient selection for DBS surgery include tremor severity, degree of refractoriness to medication, and type of tremor. In addition, individual 
patient characteristics should be considered, including age, comorbid conditions, surgical risk, patient preference, social and employment factors, and social support.

Research is ongoing to define the stimulation parameters that are most important for ensuring symptom control in patients undergoing DBS for tremor. Studies that have modeled tremor response to DBS across a range of stimulation parameters have found that suppression of tremor is most closely associated with stimulation voltage and frequency, with pulse width producing less of an effect. ${ }^{5}$ Figure 2 shows tremor power (measured in decibel units) associated with different combinations of frequency and pulse width applied to the VIM in nine patients with essential tremor. ${ }^{5}$ The observations from this study suggest that stimulation programming is complex even for essential tremor, a condition for which programming is generally among the simplest to perform.

\section{DEEP BRAIN STIMULATION FOR DYSTONIA}

Dystonia is characterized by involuntary twisting muscle contractions causing abnormal postures sometimes accompanied by jerky or repetitive involuntary movements. It may be classified according to the body part affected as generalized, segmental, or focal; in some cases it may be classified as multifocal dystonia or hemidystonia. Dystonia is also classified as primary or secondary, according to etiology. Primary dystonias are those not caused by any other identifiable condition and not associated with other neurologic abnormalities. These include idiopathic and some genetic dystonias, such as the DYT1 torsinA gene mutation. DBS of the globus pallidus internus (GPi) or subthalamic nucleus (STN) was approved by the US Food and Drug Administration under a humanitarian device exemption in 2003 for the treatment of primary generalized dystonia (PGD) in patients aged 7 years and older; GPi is the more common target). ${ }^{1}$

\section{Evidence of efficacy}

Several clinical studies have demonstrated the efficacy of DBS for patients with disabling PGD that is unresponsive to pharmacotherapy.

Long-term efficacy. Isaias and colleagues examined long-term safety and efficacy of DBS in 30 consecutive patients with PGD who were followed for at least 3 years after pallidal DBS surgery. ${ }^{6}$ DBS was delivered bilaterally in 28 patients and unilaterally in 2 patients. Clinical rating scales of motor function improved by a mean of $82.5 \%$ after 2 years, and dystonia-related disability improved by a mean of $75.2 \%$. Improvement in motor function from baseline was noted for all 30 subjects. In five patients who were followed for 7 years, improvement in motor function remained greater than $80 \%$ at the last follow-up visit. Transient regressions were noted for patients with hardware failures or whose bat-

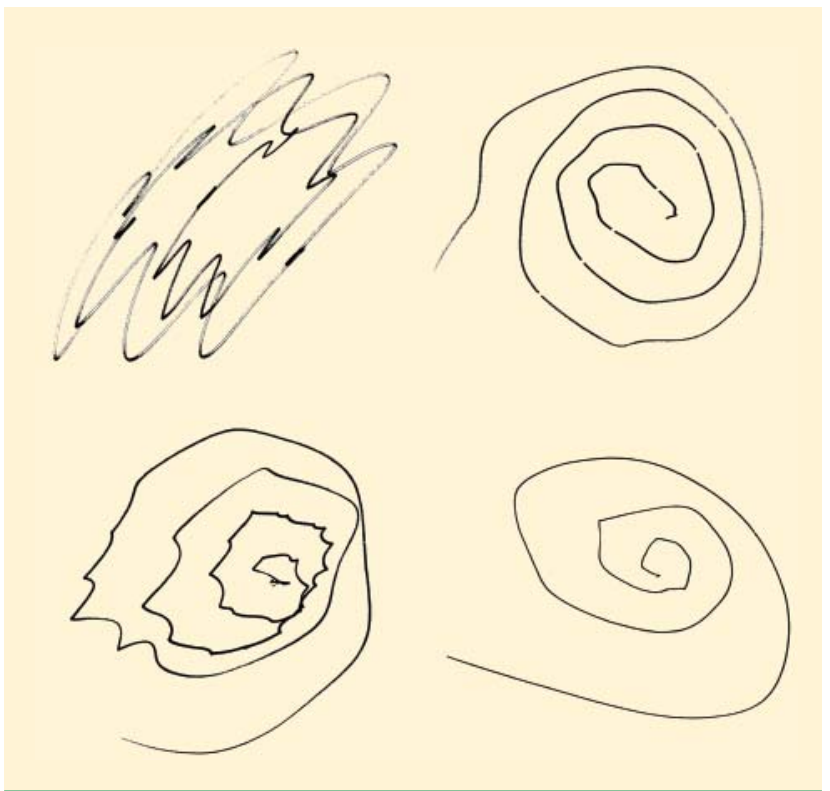

FIGURE 1. Demonstration of a tremor patient's ability to perform a drawing test before and after deep brain stimulation.

teries had reached the end of life. Stimulation-related adverse events were reported for three patients and included speech difficulties and, in one patient, transient blepharospasm.

Vidailhet and colleagues examined the efficacy of bilateral pallidal stimulation in 22 patients with PGD who were followed prospectively for 3 years. ${ }^{7}$ Mean improvement from baseline in motor function on a dystonia rating scale was $51 \%$ after 1 year and $58 \%$ after 3 years $(P=.03)$. Significant improvement was noted for individual ratings of upper and lower limb function scores. Health-related quality of life was also significantly improved at 3 -year follow-up $(P=.05)$. Serious adverse events were reported for three patients, including two lead fractures and one infection.

Results from double-blind trial. Kupsch and colleagues performed a randomized, double-blind clinical trial comparing pallidal DBS versus device implantation and sham stimulation in 40 patients with primary segmental or generalized dystonia. ${ }^{8}$ After 3 months, the mean change from baseline in severity of dystonia was $15.8 \%$ for patients who received DBS versus $1.4 \%$ with sham stimulation $(P<.001)$. At the conclusion of the double-blind treatment phase, patients entered an open-label extension phase in which all patients received DBS for another 3 months. The initial benefit of treatment was sustained across the entire 6-month study period for patients initially randomized to DBS, whereas patients who were initially randomized to sham stimulation exhibited improved motor function during the open-label extension phase. Ratings of disability and quality of life also improved for patients receiving 


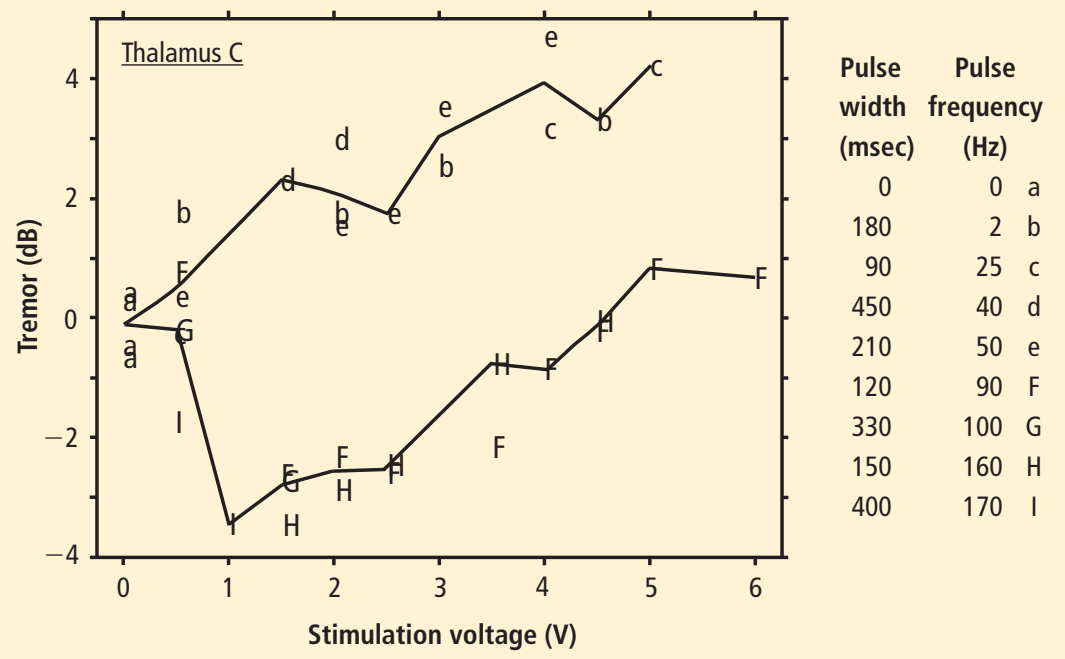

FIGURE 2. The upper curve (labeled with lower-case letters) shows various combinations of pulse width (in microseconds) and pulse frequency for frequencies less than $90 \mathrm{~Hz}$. The lower curve (labeled with upper-case letters) shows combinations of pulse width and frequency for frequencies of $90 \mathrm{~Hz}$ or greater. Each lettered point represents a frequency-pulse-width combination. Points fell into two clusters that were dependent on stimulation frequency but not pulse width. For low-frequency stimulation (upper curve), tremor increased with increasing voltage. At higher stimulation frequencies (lower curve), tremor was related to voltage in a U-shaped function. Tremor decreased as voltage increased to approximately 2 volts, and then worsened at higher voltages. ${ }^{5}$

Reprinted with permission from Journal of Clinical Neurophysiology (Cooper SE, et al. A model predicting optimal parameters for deep brain stimulation in essential tremor. J Clin Neurophysiol 2008; 25:265-273). Copyright @ 2008 by the American Clinical Neurophysiology Society.

\section{Patient selection}

Appropriate patients for DBS include those with an unequivocal diagnosis of dystonia and significant disability. Etiology and type of dystonia should also be considered. Patients with secondary dystonia (eg, due to structural brain lesions or heredodegenerative disorders) generally do not respond to DBS as well as patients with primary dystonias. A possible exception is tardive dystonia, which is caused by past exposure to dopamine receptor-blocking drugs. Although it is a secondary dystonia, tardive dystonia may respond well to DBS. Data on this point remain limited. Moreover, with tardive dystonia (as well as Sydenham chorea and poststroke hemiballismus), there may be spontaneous remission. DBS in these conditions should therefore be considered when enough time has elapsed that the likelihood of spontaneous remission is low. ${ }^{1}$

Not all dystonic symptoms have been shown to respond equally to DBS. Evidence of effectiveness is stronger and more consistent for limb and axial dystonia than for dystonic

DBS at the end of the 6-month study. Adverse events included dysarthria (five patients), serious infections (four patients), and lead dislodgement (one patient).

Response with DYT1 mutation. Coubes and colleagues examined the long-term efficacy and safety of bilateral DBS in 31 children and adults with PGD. ${ }^{9}$ PGD is associated with autosomal DYT1 mutations in approximately 30\% of cases, and these authors examined the effects of treatment in patients with and without the DYT1 mutation. After 2 years of treatment, mean scores on a dystonia clinical rating scale decreased by $79 \%$ from baseline, and mean disability ratings decreased by $65 \%$. The improvement in clinical dystonia rating scale scores was significantly greater for children than adults after 2 years $(84.7 \%$ vs $70.1 \%$; $P=$ .04). In children, functional improvement was greater after 2 years in the subset of patients with DYT 1 mutations than in the subset of patients without $(76.1 \%$ vs $44.5 \% ; P=.03)$, whereas in adults, DYT1 mutation status did not significantly influence response to treatment. One case of unilateral infection was noted, which required removal of the implant with successful reimplantation 6 months later. No other adverse events were reported. impairment of speech and swallowing. Phasic dystonia (jerky or rhythmic movements) appears to respond better than fixed postures. A critical point is that fixed postures not caused by electrically active muscle contraction will not respond to DBS. For example, bony deformity of the spine, joint disease, or tendon shortening cannot be expected to improve with DBS. The situation is complicated, since such conditions may develop as secondary consequences of dystonia. The potential for their development may warrant earlier rather than later DBS surgery in childhood-onset PGD. ${ }^{10}$

\section{UNRESOLVED ISSUES IN DBS FOR DYSTONIA}

\section{How aggressively should other therapies be tried before starting DBS?}

Pharmacologic options include a range of oral, intramuscular, and intrathecal agents. Injection of botulinum toxin to denervate affected muscles is a mainstay of treatment for focal or segmental dystonia, but often fails to improve symptoms because of the involvement of a large number of muscles, complexity of the movement pattern, or the development of neutralizing antibodies. ${ }^{8}$ With the exception of levodopa-responsive PGD, other 
pharmacologic therapy for PGD is generally of limited effectiveness for controlling symptoms of dystonia. ${ }^{9}$ Oral or intrathecal baclofen may improve symptoms, but often produces unacceptable sedation.

\section{How important is intraoperative microelectrophysiology?}

Although contemporary imaging techniques are important in the correct placement of stimulating electrodes, the available techniques do not always provide sufficient resolution to delineate the STN or GPi. The accuracy of electrode placement may also be influenced by distortions caused by lack of homogeneity among magnetic resonance images, brain shift, and signal deflections from cannulae or electrodes. ${ }^{14}$ These errors may result in significant deviation of electrode placement from the intended target. Microelectrode recording and microstimulation may be used to map the target region and refine the surgical target. It is widely, but not universally, held that this strategy contributes to superior accuracy and outcomes; it ordinarily requires an awake procedure, which is not always feasible in patients with severe dystonia or in pediatric patients. ${ }^{11}$

\section{How should be programming (stimulator adjustment) be performed?}

Research continues to refine our understanding of how electrical parameters such as voltage, frequency, and pulse width affect clinical outcomes in patients undergoing DBS for dystonia. Some programming approaches, such as long pulse width and high frequency, that were once generally accepted are now widely questioned. Another major unresolved question is: "How long should it take to see the results of stimulation?" In the clinical studies described above, continued improvement was generally observed over months or even years, and, in most patients, stimulators are incrementally adjusted over an extended period. However, some patients may experience much more rapid onset of benefit.

\section{Long-term DBS management of dystonia}

Unlike DBS for Parkinson disease or even essential tremor, DBS for dystonia is performed in young patients. This creates special challenges in pediatric patients, who can be expected to grow and develop after device implantation. As a result, children may require additional surgeries to reposition devices.

In addition, the most widely used devices require repeated battery replacement surgeries, although newer rechargeable devices are becoming available.

Finally, there is a nontrivial incidence of hardwarerelated complications when devices are used continuously for many years. Although individual dystonia patients vary in the acuity of their response to the cessation of stimulation, ${ }^{6}$ deterioration can be acute and dramatic. In long-term studies of bilateral pallidal stimulation described above, hardware failures were the most commonly reported adverse events, including unilateral or bilateral lead fracture. ${ }^{7,9}$ These appear to be more frequent in patients with dystonia than in other movement disorders.

\section{SUMMARY AND CONCLUSIONS}

Deep brain stimulation produces marked and longlasting improvement in motor function and disability in patients with hyperkinetic disorders. In patients with essential tremor, stimulation usually targets the VIM of the thalamus. Reduction in tremor is most closely related to stimulation frequency and voltage, whereas pulse width has little effect on treatment outcome. In patients with dystonia, stimulation typically targets the GPi or STN. Long-term prospective clinical trials demonstrated significant reductions in motor severity rating scale scores. Selecting patients for DBS requires careful consideration of a range of factors, including the specific clinical presentation, treatment history, and social support. Areas of current investigation include optimal stimulation programming, intraoperative mapping, and the long-term efficacy and safety of stimulation.

\section{REFERENCES}

1. Montgomery EB Jr. Deep brain stimulation for hyperkinetic disorders. Neurosurg Focus 2004; 17:E1.

2. Deuschl G, Bain P, Brin M. Consensus statement of the Movement Disorder Society on tremor. Ad Hoc Scientific Committee. Mov Disord 1998; 13(suppl 3):2-23.

3. Lyons KE, Pahwa R. Deep brain stimulation and tremor. Neurotherapeutics 2008; 5:331-338.

4. Koller WC, Lyons KE, Wilkinson SB, Pahwa R. Efficacy of unilateral deep brain stimulation of the VIM nucleus of the thalamus for essential head tremor. Mov Disord 1999; 14:847-850.

5. Cooper SE, Kuncel AM, Wolgamuth BR, Rezai AR, Grill WM. A model predicting optimal parameters for deep brain stimulation in essential tremor. J Clin Neurophysiol 2008; 25:265-273.

6. Isaias IU, Alterman RL, Tagliati M. Deep brain stimulation for primary generalized dystonia: long-term outcomes. Arch Neurol 2009; 66:465-470.

7. Vidailhet M, Vercueil L, Houeto JL, et al. Bilateral, pallidal, deepbrain stimulation in primary generalised dystonia: a prospective 3 year follow-up study. Lancet Neurol 2007; 6:223-229.

8. Kupsch A, Benecke R, Müller J, et al. Pallidal deep-brain stimulation in primary generalized or segmental dystonia. N Engl J Med 2006; 355:1978-1990.

9. Coubes P, Cif L, El Fertit H, et al. Electrical stimulation of the globus pallidus internus in patients with primary generalized dystonia: long-term results. J Neurosurg 2004; 101:189-194.

10. Loher TJ, Capelle HH, Kaelin-Lang A, et al. Deep brain stimulation for dystonia: outcome at long-term follow-up. J Neurol 2008; 255:881-884.

11. Lozano AM, Snyder BJ, Hamani C, Hutchison WD, Dostrovsky JO. Basal ganglia physiology and deep brain stimulation. Mov Disord 2010; 25(suppl 1):S71-S75.

Correspondence: Scott Cooper, MD, PhD, Center for Neurological Restoration, Cleveland Clinic, 9500 Euclid Avenue, U2, Cleveland, OH 44195; coopers2@ccf.org 
Downloaded from www.ccjm.org on April 26, 2023. For personal use only. All other uses require permission. 